\title{
Microsurgical Treatment of Tuberculum Sellae Meningiomas with Visual Impairments: A Chinese Experience of 56 Cases
}

\author{
Hui ZHOU ${ }^{1,2}$, Zhen WU', Liang WANG ${ }^{1}$, Junting ZHANG ${ }^{1}$ \\ ${ }^{1}$ Capital Medical University, Beijing Tiantan Hospital, Department of Neurosurgery, Beijing, China \\ ${ }^{2}$ Xuzhou Medical College, Lianyungang Hospital, Department of Neurosurgery, Lianyungang, Jiangsu Province, China
}

\section{ABSTRACT}

\begin{abstract}
AIM: Tuberculum sellae meningiomas (TSMs) are suprasellar lesions that commonly extend to the medial side of the optic nerve, resulting in visual impairments in the affected eye as the initial and most common symptoms. The primary goal of surgical treatment for TSM is the preservation or improvement of visual function. The aim of the present study was to assess the clinical outcomes of TSMs treated with microsurgery at our center.
\end{abstract}

MATERIAL and METHODS: A retrospective analysis was performed on 56 patients with TSMs associated with visual impairments who visited our neurosurgery center between January 2008 and January 2012. These patients underwent microsurgery using the unilateral subfrontal approach $(n=22)$, the lateral frontal base approach $(n=28)$, or the frontotemporal approach $(n=6)$. Outcomes were complete tumor removal, improvements of visual impairments and survival to the surgery. After surgery, patients were classified according to Simpson classification: Grade I in 22 cases, grade II in 28 cases, and grade IV in 6 cases.

RESULTS: Postoperatively, visual impairments were improved in 47 cases, unchanged in 7 cases, and worsened in 2 cases. There was no patient's death.

CONCLUSION: TSMs are operated in China with favorable outcomes.

KEYWORDS: Meningioma, Microsurgery, Sella turcica, Visual disorders

\section{INTRODUCTION}

Meningiomas represents about $33 \%$ of all brain tumors, and tuberculum sellae (TS) meningiomas (TSM) represent about $5-10 \%$ of meningiomas, with an estimated prevalence of 12,750 cases in the United States $(1,12,14,22)$. TSMs arise from the dura of the TS, the chiasmatic sulcus, the limbus sphenoidale, the sphenoid planum, or the diaphragma sellae. Because of the anatomic proximity of the lateral end of the TS to the optic canal, the tumor tends to extend into the optic canal underneath the nerve. As the tumor grows, it compresses the optic nerve, the internal carotid artery, the pituitary and the pituitary stalk $(11,19)$, resulting in the typical clinical feature of TSMs, i.e. primary optic atrophy with bitemporal hemianopsia. Visual impairments in the affected eye are the initial and most common symptoms of TSMs $(3,11)$.
Therefore, the goal of the surgical treatment for TSM is the total resection of the tumor and the preservation or improvement of the visual function (2). However, because of the proximity to important anatomic structures, the surgery of these TSMs is complex. Surgical approaches for TSMs include the bifrontal approach, the unilateral subfrontal approach, the frontotemporal pterional approach, the orbitocranial (or orbitozygomatic) approach, the combined extradural and intradural approach, and the transsphenoidal approach $(1,5,6,13)$.

There is a lack of data about the treatment of TSMs in China. Therefore, the aim of this retrospective study was to analyze 56 consecutive cases of surgically resected TSMs since 2008 in our institute (China). Total resection of the tumor and improvement of postoperative visual function were assessed as primary outcomes. 


\section{MATERIAL and METHODS}

\section{Subjects}

This retrospective study was carried out at the skull base department of neurosurgery center in Beijing Tiantan Hospital, Capital Medical University. A total of 56 consecutive TSM patients with visual impairments were enrolled in the present study, from January 2008 to January 2012. Patients had to suffer from visual impairment and to have an imagery-based diagnosis of TSM. The present study was approved by our ethical committee, and an individual consent was waived.

\section{Imaging}

All patients underwent imaging examinations including computed tomography (CT) and magnetic resonance imaging (MRI), as well as eye examinations in the immediate preoperative and postoperative period. Gadolinium-enhanced $\mathrm{MRI}$ and magnetic resonance angiography (MRA) were used to show the relationship between the tumor and the cerebral arteries, the optic nerve, the pituitary stalk and bilateral cavernous sinuses. Gadolinium-enhanced T1 axial MRI was used to show the potential encasing of the internal carotid arteries. Gadolinium-enhanced T1 sagittal MRI was used to determine if the mass had a dural base on the planum sphenoidale, chiasmatic sulcus, and TS, and if the sella turcica was not enlarged. Gadolinium-enhanced T1 coronal MRI was used to show the relationship between the tumor and the internal carotid arteries, middle cerebral arteries, and anterior cerebral arteries on both sides of the mass. T2weighted imaging (T2WI) was used to show isointense or hyperintense signal intensity, which could reveal the edema surrounding the tumor.

\section{Surgical Approach}

The surgery was performed using the unilateral subfrontal, the lateral frontal base approach or the frontotemporal approach, according to the tumors' size, location, growth pattern and adjacent relationship to important structures $(2,8,12)$. These three methods are the only ones used in our hospital. The unilateral subfrontal approach was used for medium tumors mainly located in the midline with a wide base and bilateral growth. The lateral frontal base approach was used for some medium tumors and small tumors, mostly growing unilaterally. The frontotemporal approach was used for the large tumors and the medium tumors growing from the midline to the oneside anterior clinoid process.

The rotation angle of the head to the contralateral side varied according to the surgical approach $\left(30^{\circ}\right.$ head rotation for the unilateral subfrontal approach, and $30-45^{\circ}$ head rotation for the lateral frontal base approach and the frontotemporal approach).

The incision approaching the hairline arc, progressing ahead and passing through the midline was different according to the surgical approach (to the lateral superior temporal line for the unilateral subfrontal approach, and about $2 \mathrm{~cm}$ over the midline for the lateral frontal base approach and the frontotemporal approach). The craniotomy was performed on the lateral frontal bone and the temporalis muscle was stripped from the superior temporal line and retracted to the lateral side. An incision of the temporalis muscle and retraction to the temporal base is required for the frontotemporal craniotomy. After entering the skull, the sphenoid ridge was slicked for the frontotemporal craniotomy, to ensure the exposure of the bilateral blood vessels of the Sylvian fissure.

If the ipsilateral optic nerve was closely adhering to the tumor, the arachnoid membrane gap outside the tumor was separated through the second gap, and the tumor underneath the optic nerve was dissociated sharply. If MRI showed that the tumor invaded the optic canal, considering that the tumor vessels underneath the shuttle of the dura mater of the optic canal are in a blind spot, the dura mater of the skull base at the medial anterior clinoid process was cut with a sharp knife and was shrunk using the bipolar coagulation tool or removed sharply. After devascularization, the tumor was detached and removed pieces by pieces. The hyperplastic dura mater on the tumor vessels was removed. In the present study, there was no case of drilling into the sphenoid sinus. The bone was ground, and bone wax was routinely applied. The grinding surface was smoothed with a small piece of cottonoid.

\section{Follow-up}

All 56 patients were followed-up regularly by the Neurosurgery and Ophthalmology Departments at 3-month intervals. The follow-up period varied from 1 to 36 months (mean follow-up time duration of $27.47 \pm 7.22$ months). Visual examinations and $\mathrm{MRI}$ were performed during follow-up.

\section{Outcomes}

Outcomes were the complete removal of the tumor, improvements in visual function according to the visual grading system (2), and survival to the surgery.

\section{Statistics}

Only descriptive statistics were used in the present study.

\section{RESULTS}

\section{Patients' Characteristics}

Table I shows the patients' characteristics. Fifty-six patients were included (26 males and 30 females), with a mean age of 42.5 years (range: 21 to 69 years). The duration of disease ranged from 1 month to 4.3 years. The median duration of visual impairment symptoms was 14 months. There were 34 cases $(60.7 \%)$ of monocular visual impairments, and 22 cases (39.3\%) of binocular visual impairments, including 4 cases (7.1\%) complicated by monocular blindness. Visual field defect was detected in 31 cases (55.4\%). There were 25 cases (44.6\%) of vision decrease without changes in visual field, and 2 cases (3.6\%) of visual field defect without vision decrease. In accordance with the visual grading system (grade $1:<0.1$ to 0.05 ; and grade 2 : $<0.3$ to 0.1 (2), 22 patients (39.3\%) had low vision under grade 2 , and 34 patients (60.7\%) had low vision more than grade 2 . Fundus examination showed 36 cases $(64.3 \%)$ of optic atrophy, including 16 cases $(28.6 \%)$ of bilateral optic atrophy and 20 cases $(35.7 \%)$ of unilateral optic atrophy; 16 cases (28.6\%) with unilateral or bilateral edema of 
the optic papilla; and 4 cases $(7.1 \%)$ with normal optic papilla.

\section{Imagery}

Figure $1 \mathrm{~A}-\mathrm{C}$ shows a typical preoperative MRI showing that the tumor, as a round or oval space-occupying lesion, was located in the suprasellar area. MRI revealed that the lesions were uniformly enhanced and that a "dural tail" sign was seen in most lesions.

\section{Tumors' Characteristics}

In this present study, 15 patients had tumors growing to the lateral and upper lateral side, pushing and wrapping around the optic nerve and the cerebral arteries; 22 cases with tumors compressing downward the optic nerve and optic chiasm; and 19 cases with tumors compressing upward and outward the optic nerve and optic chiasm, including 8 cases with tumors growing to the saddle back and compressing the pituitary stalk and hypothalamus. Maximum tumor diameter was large $(>5 \mathrm{~cm})$ in 6 cases, medium $(3$ to $5 \mathrm{~cm})$ in 26 cases, and small $(<3 \mathrm{~cm})$ in 24 cases (Table II).

\section{Surgeries' Characteristics}

Table II shows the surgeries' characteristics and Figure 2 shows a TSM during surgery. Simpson Grade I resection (total resection of tumor and hyperplastic dura mater and grinding of hyperplastic skull) was performed in 22 cases, Grade II resection (total resection of tumor and fulguration of tumor basal dura mater) was performed in 28 cases, and subtotal resection (residual tumors were observed around important cerebral arteries and optic nerves under a microscope) was performed in 6 cases, including 3 cases undergoing postoperative radiation therapy. The rate of total tumor resection was therefore $89.3 \%$. Figure 3 shows a typical case after removal of the tumor.

\section{Complications}

Postoperative complications included fever in 8 cases, cerebrospinal fluid (CSF) rhinorrhea in 2 cases, electrolyte

imbalance in 9 cases, and transient diabetes insipidus in 12 cases. All complications improved after postoperative treatment. There were no operative deaths (Table II).

Table I: Patients' Characteristics

\begin{tabular}{|c|c|}
\hline Characteristic & Value \\
\hline \multicolumn{2}{|l|}{ Gender } \\
\hline Male & $26(46.4 \%)$ \\
\hline Female & $30(53.6 \%)$ \\
\hline Age, mean (range) & $42.5(21-69)$ years \\
\hline Disease duration & 1 month-4.3 years \\
\hline Duration of symptoms, median & 14 months \\
\hline \multicolumn{2}{|l|}{ Visual impairments } \\
\hline Monocular & $34(60.7 \%)$ \\
\hline Binocular & $22(39.3 \%)$ \\
\hline Monocular blindness & $4(7.1 \%)$ \\
\hline Visual defect & $31(55.4 \%)$ \\
\hline Vision decrease alone & $25(44.6 \%)$ \\
\hline Visual field defect alone & $2(3.6 \%)$ \\
\hline Low vision under grade 2 & $22(39.3 \%)$ \\
\hline Low vision above grade 2 & $34(60.7 \%)$ \\
\hline Optic atrophy & $36(64.3 \%)$ \\
\hline Unilateral & $20(35.7 \%)$ \\
\hline Bilateral & $16(28.6 \%)$ \\
\hline Edema of the optic papilla & $16(28.6 \%)$ \\
\hline Normal optic papilla & $4(7.1 \%)$ \\
\hline
\end{tabular}
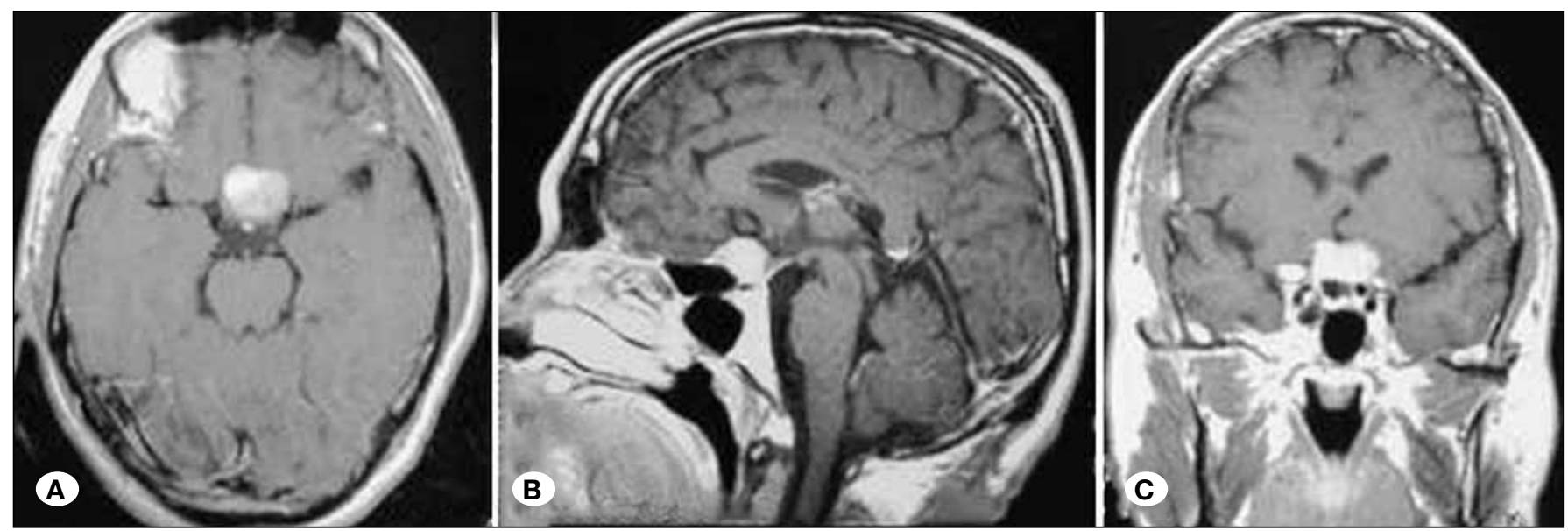

Figure 1: A 49-year-old man had a $23 \mathrm{~mm}$ meningioma that had been removed totally through a right lateral frontal base approach. Preoperative enhanced MRI of TSM showed the tumor located at tuberculum sella, predominantly medial to optic nerve. A) The meningioma was located in the sellar region (axial image). B) The meningioma compressed the optic chiasma (sagittal image).

C) The meningioma was close to the bilateral internal carotid artery (coronal image). 
Table II: Tumors' and Surgeries' Characteristics

\begin{tabular}{lc}
\hline \multicolumn{1}{l}{ Characteristic } & Value \\
\hline \multicolumn{1}{l}{ Tumor size } & $6(10.7 \%)$ \\
\hline$>5 \mathrm{~cm}$ & $26(46.4 \%)$ \\
\hline $3-5 \mathrm{~cm}$ & $24(42.9 \%)$ \\
\hline$<3 \mathrm{~cm}$ & \\
\hline Resection & $22(39.3 \%)$ \\
\hline Simpson Grade I & $28(50.0 \%)$ \\
\hline Simpson Grade II & $6(10.7 \%)$ \\
\hline Subtotal & $89.3 \%$ \\
\hline Total tumor resection rate & $8(14.3 \%)$ \\
\hline Postoperative complications & $2(3.6 \%)$ \\
\hline Fever & $9(16.1 \%)$ \\
\hline CSF rhinorrhea & $12(21.4 \%)$ \\
\hline Electrolyte imbalance & 0 \\
\hline Transient diabetes insipidus & $83.9 \%$ \\
\hline Death & \\
\hline Visual impairment improvement rate & \\
\hline
\end{tabular}

CSF: cerebrospinal fluid.

\section{Visual Improvements}

Visual impairments improved in 47 cases after surgery, with an improvement rate of $83.9 \%$. No obvious changes were observed in 7 cases, while aggravated visual impairments were detected in 2 cases. Although transient deteriorating visual impairments were observed on the operated side of some patients after surgery, most patients recovered at different degrees with elapsing time.

\section{Follow-up}

All 56 subjects were followed up for 1 to 36 months. Figure $4 \mathrm{~A}-\mathrm{C}$ shows typical MRI 3 months after surgery. During follow-up, of the 34 patients with preoperative monocular visual impairments, postoperative improvement occurred in 30 cases and no obvious changes were observed in 4 cases. Among the 22 cases with binocular vision disorders, all cases had improved postoperative vision at various degrees on the less severe side, and 2 cases had deteriorated postoperative vision in the more severe side. Of the 4 cases with monocular blindness, no obvious changes were observed in 3 cases after surgery, and perception of light was present in 1 case after surgery, which was thought to be related to the large tumor volume and long duration of optic nerve compression.

\section{DISCUSSION}

TSMs are suprasellar lesions that commonly extend to the medial side of the optic nerve, resulting in visual impairments

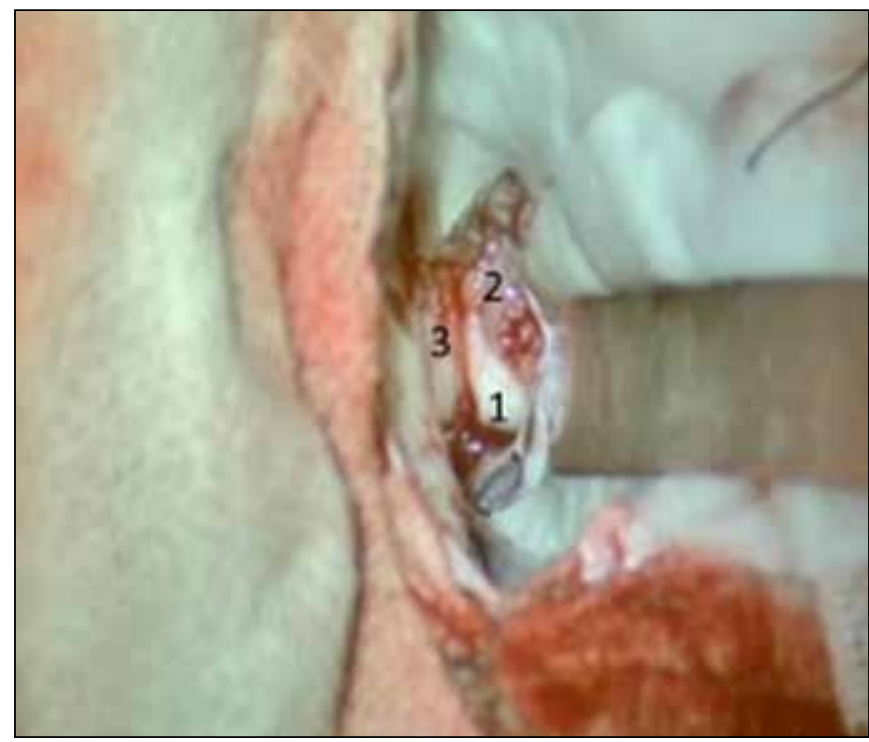

Figure 2: The perioperative view was from the same patient as that in Figure 1. 1. Ipsilateral optic nerve. 2. The tumor. 3. Base of the TSM.

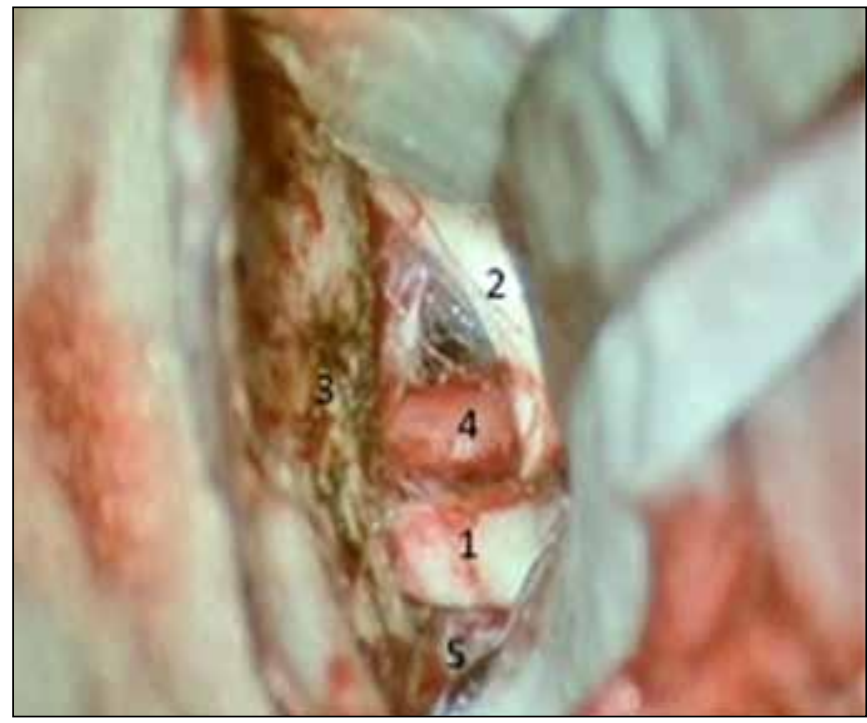

Figure 3: The perioperative view was from the same patient as that in Figure 2, after tumor resection. 1. Ipsilateral optic nerve. 2. Contralateral optic nerve. 3. Base of the TSM. 4. Pituitary stalk. 5. Ipsilateral internal carotid artery.

in the affected eye as the initial and most common symptoms. The primary goal of surgical treatment for TSM is preservation or improvement of visual function. The aim of the present study was to assess the clinical outcomes of TSMs treated with microsurgery in a Chinese center.

In the present study, complete tumor resection was performed in 50 cases, and subtotal resection in 6 , resulting in a complete tumor resection rate was $89.3 \%$. After operation, the visual improvement rate was $83.9 \%$. Few adverse events were observed and were successfully treated. There was no patient's death. 

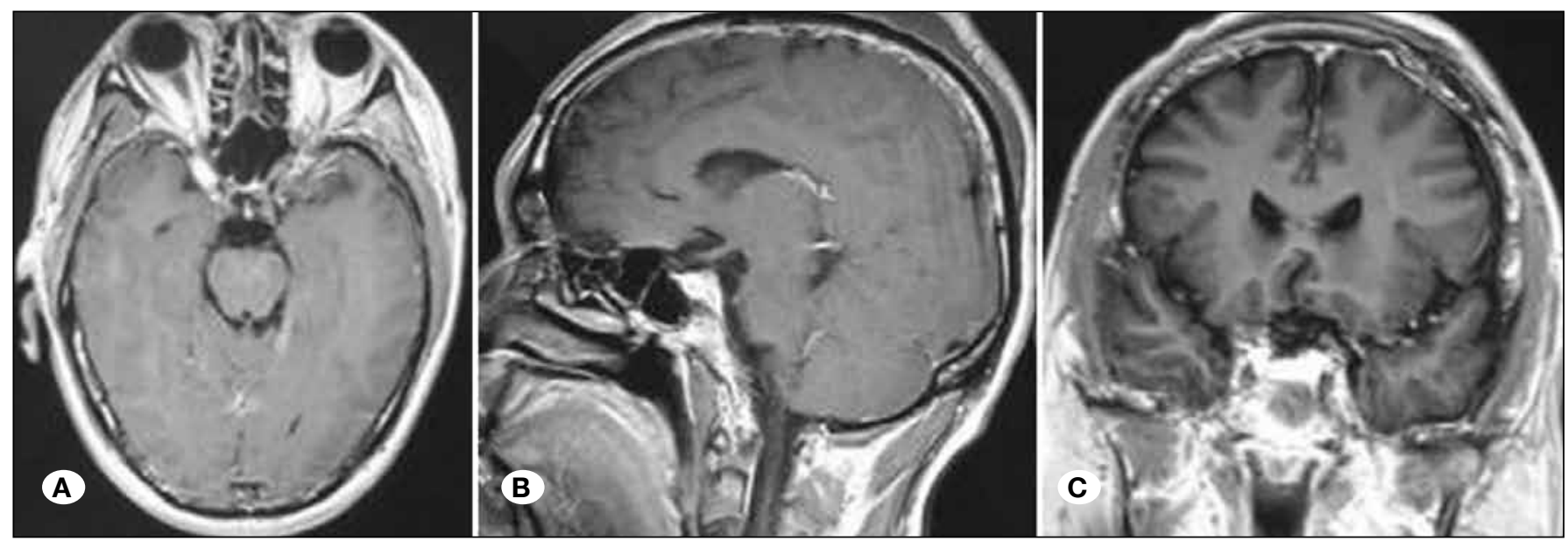

Figure 4: The postoperative images were from the same patient as that in Figure 1. Enhanced MRI at 3 months after surgery (a-c). A) The TSM was completely removed (axial image). B) The pituitary stalk returned to its normal position (sagittal image). C) The meningioma between bilateral internal carotid arteries was completely removed (coronal image).

The goals of TSMs surgery are: 1) complete excision of the tumor with the dural attachment; and 2) preservation of the surrounding neurovascular structures, especially the optic nerves and cerebral arteries. The main way to improve patients' symptoms is to use microsurgery for adequate optic nerve decompression. Therefore, the postoperative recovery of visual function has become an important standard for assessing the success of surgery for $\operatorname{TSMs}(8,12,18,21)$.

The surgical approach has been shown to have a clear impact on visual improvement $(15,20)$. It is reported that the mean rate of visual impairment improvement was $68.8 \%$ using the frontotemporal pterional approach (17). A previous study using the frontolateral or the fronto-orbital approach showed visual improvements in $64.9 \%$ of TSM cases, and no cases of visual deterioration (16). Studies were performed to compare the extended transsphenoidal surgery (ETSS) and the transcranial surgery (TCS) approaches, and showed that $71-81 \%$ of patients treated with ETSS had a visual improvement vs. 59$61 \%$ using TCS $(7,15)$. The unifrontal approach showed an improvement rate of $70 \%$ (10), while the bilateral subfrontal approach showed an improvement rate of $90.6 \%$ (4). Using the pterional approach, the visual improvement rate was particularly low, at $53.2 \%$ (3). In the present study, using the unilateral subfrontal, the lateral frontal base approach or the frontotemporal approach, 47 TSM patients (83.9\%) with visual impairment had an improved postoperative vision, and two cases had deteriorated postoperative visual impairment. In these two patients, the tumor was huge $(>5 \mathrm{~cm})$ and the tumor texture was hard. Of these two cases, one had light perception before surgery, and optic nerve was found to be compressed by the tumor and to be adherent to the tumor without arachnoid membrane interface. During tumor resection, the optic nerve suffered from injuries and impaired blood supply. Moreover, the large tumor size, the long disease duration, and the resulting long-term compression on the optic nerve may be responsible for the deteriorated postoperative visual impairments, as previously observed $(8,20,21)$. It was observed that the presence of the arachnoid membrane interface is of particular importance for the recovery of postoperative vision $(8,20,21)$. It was reported that the importance and duration of the visual impairment, the tumor size and vascular adherences were factors influencing the surgical success rate (9), as well as a thin atrophic optic nerve, nerve encasement by the tumor and tumor adhesion (3).

The rate of complete tumor removal seems to be dependent upon the surgical approach. Indeed, in a previous study, $92.4 \%$ of TSMs were totally resected using the frontolateral or the fronto-orbital approach (16). A study using the unifrontal approach showed a total tumor resection rate of $84.2 \%(10)$, while another study using the bilateral subfrontal approach showed a total resection rate of $79.4 \%$ (4). The success rate using TCS was reported to be $86.4 \%$ and to be $83.3 \%$ using ETSS (7). The pterional approach showed a success rate of $90.3 \%$ (3). In the present study, using the unilateral subfrontal, the lateral frontal base approach or the frontotemporal approach, Simpson grade I or II resection was achieved in $89.3 \%$ of patients.

TSM surgery is performed near the sinuses, and there is a risk of sinus perforation leading to CSF rhinorrhea and to a risk of meningitis $(2,12,21)$. A previous study showed a postoperative comorbidity rate of $16.1 \%$, including seizures, and impaired mental functions (3). Another study observed CSF rhinorrhea, infarction, seizures and transient diabetes insipidus, but no mortality (16). In the present study, the 2 cases of cerebrospinal fluid rhinorrhea were both caused by the unilateral subfrontal approach. The most frequent complication was diabetes insipidus. No patient dies from the surgery.

The present study has several limitations. First, it was performed in a small number of patients. Furthermore, no control or comparator group was used. In the future, we will aim toward performing a larger multicentre study to compare the different surgical approaches available to treat TSMs. 


\section{CONCLUSION}

In conclusion, using three different approaches, TSMs are operated in a Chinese center with a high surgical success rate and a high rate of improvements in vision. The rate of complications was acceptable.

\section{REFERENCES}

1. Al-Mefty O, Holoubi A, Rifai A, Fox JL: Microsurgical removal of suprasellar meningiomas. Neurosurgery 16:364-372,1985

2. Andrews BT, Wilson CB: Suprasellar meningiomas: The effect of tumor location on postoperative visual outcome. J Neurosurg 69:523-528,1988

3. Bassiouni H, Asgari S, Stolke D: Tuberculum sellae meningiomas: Functional outcome in a consecutive series treated microsurgically. Surg Neurol 66:37-44; discussion 44-45,2006

4. Chokyu I, Goto T, Ishibashi K, Nagata T, Ohata K: Bilateral subfrontal approach for tuberculum sellae meningiomas in long-term postoperative visual outcome. J Neurosurg 115:802810,2011

5. Ciric I, Rosenblatt S: Suprasellar meningiomas. Neurosurgery 49:1372-1377, 2001

6. Couldwell WT, Weiss MH, Rabb C, Liu JK, Apfelbaum RI, Fukushima T: Variations on the standard transsphenoidal approach to the sellar region, with emphasis on the extended approaches and parasellar approaches: Surgical experience in 105 cases. Neurosurgery 55:539-547; discussion 547-550,2004

7. de Divitiis E, Esposito F, Cappabianca P, Cavallo LM, de Divitiis O: Tuberculum sellae meningiomas: High route or low route? A series of 51 consecutive cases. Neurosurgery 62:556-563; discussion 556-563,2008

8. Fahlbusch R, Schott W: Pterional surgery of meningiomas of the tuberculum sellae and planum sphenoidale: Surgical results with special consideration of ophthalmological and endocrinological outcomes. J Neurosurg 96:235-243,2002

9. Galal A, Faisal A, AI-Werdany M, El Shehaby A, Lotfy T, Moharram $\mathrm{H}$ : Determinants of postoperative visual recovery in suprasellar meningiomas. Acta Neurochir (Wien) 152:69-77,2010

10. Goel A, Muzumdar D, Desai KI: Tuberculum sellae meningioma: A report on management on the basis of a surgical experience with 70 patients. Neurosurgery 51:1358-1363; discussion 13631364,2002
11. Gokalp HZ, Arasil E, Kanpolat $\mathrm{Y}$, Balim T: Meningiomas of the tuberculum sella. Neurosurg Rev 16:111-114,1993

12. Jallo GI, Benjamin V: Tuberculum sellae meningiomas: Microsurgical anatomy and surgical technique. Neurosurgery 51:1432-1439; discussion 1439-1440,2002

13. Jho HD: Endoscopic endonasal approach to the optic nerve: $A$ technical note. Minim Invasive Neurosurg 44:190-193,2001

14. Kadis GN, Mount LA, Ganti SR: The importance of early diagnosis and treatment of the meningiomas of the planum sphenoidale and tuberculum sellae: A retrospective study of 105 cases. Surg Neurol 12:367-371,1979

15. Kitano M, Taneda M, Nakao Y: Postoperative improvement in visual function in patients with tuberculum sellae meningiomas: Results of the extended transsphenoidal and transcranial approaches. J Neurosurg 107:337-346,2007

16. Li-Hua C, Ling C, Li-Xu L: Microsurgical management of tuberculum sellae meningiomas by the frontolateral approach: Surgical technique and visual outcome. Clin Neurol Neurosurg 113:39-47,2011

17. Margalit N, Kesler A, Ezer H, Freedman S, Ram Z: Tuberculum and diaphragma sella meningioma--surgical technique and visual outcome in a series of 20 cases operated over a 2.5 -year period. Acta Neurochir (Wien) 149:1199-1204; discussion 1204,2007

18. Raco A, Bristot R, Domenicucci M, Cantore G: Meningiomas of the tuberculum sellae. Our experience in 69 cases surgically treated between 1973 and 1993. J Neurosurg Sci 43:253-260; discussion 260-262,1999

19. Rhoton AL Jr: The sellar region. Neurosurgery $51: \mathrm{S} 335-374,2002$

20. Rosenstein J, Symon L: Surgical management of suprasellar meningioma. Part 2: Prognosis for visual function following craniotomy. J Neurosurg 61:642-648,1984

21. Schick U, Hassler W: Surgical management of tuberculum sellae meningiomas: Involvement of the optic canal and visual outcome. J Neurol Neurosurg Psychiatry 76:977-983,2005

22. Wiemels J, Wrensch M, Claus EB: Epidemiology and etiology of meningioma. J Neurooncol 99:307-314,2010 